\title{
Fluorescent tags to explore cell wall structure and dynamics
}

\author{
Martine Gonneau ${ }^{1,2}$, Herman Höfte ${ }^{1,2}$ and Samantha Vernhettes ${ }^{1,2}$ * \\ 1 INRA, UMR1318, Institut Jean-Pierre Bourgin, Saclay Plant Sciences, Versailles, France \\ ${ }^{2}$ AgroParisTech, Institut Jean-Pierre Bourgin, Versailles, France
}

\section{Edited by:}

Jose Manuel Estevez, University of Buenos Aires, Argentina

\section{Reviewed by:}

Staffan Persson, Max-Planck

Gesellschaft, Germany

David Ehrhardt, Stanford

University, USA

*Correspondence:

Samantha Vernhettes, Institut

Jean-Pierre Bourgin, UMR1318

INRA-AgroParisTech, Bâtiment 2,

INRA Centre de Versailles-Grignon,

Route de St-Cyr (RD10), 78026

Versailles Cedex, France. e-mail:

samantha.vernhettes@versailles.inra.fr
Plant cell walls are highly dynamic and heterogeneous structures, which vary between cell types, growth stages but also between microdomains within a single cell wall. In this review, we summarize the imaging techniques using fluorescent tags that are currently being used and which should in the coming years revolutionize our understanding of the dynamics of cell wall architecture and the cellular processes involved in the synthesis of cell wall components.

\section{Keywords: plant cell-wall, fluorescence microscopy}

\section{INTRODUCTION}

We currently have a reasonably good understanding of the structure of polysaccharides and, in large part thanks to the use of genetics, rapid progress is being made in the understanding of the mechanisms and regulation of wall synthesis. A major challenge for the future will be to understand cell wall architecture and its dynamics during growth. Here, we will review recent progress in fluorescence microscopy, which should largely contribute to reaching this goal.

\section{CELL WALL STRUCTURE AND DYNAMICS}

Plant cell walls are highly dynamic and heterogeneous structures, which vary between cell types, growth stages but also between microdomains within a single cell wall. Tools are needed to visualize this heterogeneity in living cells with a high spatial and temporal resolution.

Electron microscopy techniques [transmission electron microscopy (TEM) and field emission scanning electron microscopy (FESEM)] have been instrumental in obtaining information on wall architecture. However, these techniques require fixed material, which is not always representative for native hydrated cell walls and provide little or no information on cell wall

\footnotetext{
Abbreviations: BiFC, bimolecular fluorescence complementation; GFP, green fluorescent protein; FESEM, field emission scanning electron microscopy; FLIP, fluorescence loss in photobleaching; FPALM, fluorescence photoactivated localization microscopy; FRAP, fluorescence recovery after photobleaching; FRET, fluorescence resonance energy transfer; LSCM, laser scanning confocal microscopy; LSFM, light-sheet-based fluorescence microscopy; PALM, photoactivated localization microscopy; SDCM, spinning disk confocal microscopy; SIM, structured illumination microscopy; SPIM, selective plane illumination; STED, stimulated emission depletion; STORM, stochastic optical reconstruction microscopy; TEM, transmission electron microscopy; TIRFM, total internal reflection fluorescence microscopy; VAEM, variable-angle epifluorescence microscopy; YFP, yellow fluorescent protein.
}

dynamics. The use of fluorescent probes should provide access to dynamic aspects. A large number of anti-glycan antibodies are available (Knox, 2008; Moller et al., 2008; Pattathil et al., 2010; Ralet et al., 2010, see the review Lee et al., 2011; PlantProbes ${ }^{1}$; CarboSource Services ${ }^{2}$ ). In addition, the increasing number of sequenced genomes of plants and microorganisms provide a virtually unlimited supply of often highly specific carbohydrate binding domains (Guillen etal., 2010). These probes in general provide a static picture but labeling of living cells is also possible. For instance, Domozych et al. (2009) used antibodies to study pectin maturation in the single celled Charophycean alga Penium margaritaceum. It will be interesting to explore similar approaches to study wall dynamics in higher plants. Caution is required in using these probes since polysaccharide epitopes are frequently masked by other polymers (Marcus et al., 2008, 2010).

Recently, significant progress was also made in the visualization of cellulose dynamics in living cells using the cellulose-specific dye Pontamine Fast Scarlet 4B (S4B; Anderson et al., 2010). Confocal microscopy of S4B-stained roots revealed the orientation of cellulose microfibrils. Interestingly, the staining did not interfere with growth and a rotation of the microfibrils, from transverse to longitudinal to the elongation axis, could be observed during cell elongation. This is the first in vivo validation of the 57-year-old "multinet growth" hypothesis (Roelofsen and Houwink, 1953).

Enzyme activity can also be visualized in the wall using fluorescent donor substrates. Sulforhodamine conjuguates of xyloglucan oligosaccharides (XGO-SRs) were used to visualize endogenous xyloglucan endo-transglycosylase (XET) activity (of the xyloglucan transglycosylase/hydrolase enzyme, XTH) in Arabidopsis and

\footnotetext{
${ }^{1}$ http://www.plantprobes.net/

${ }^{2}$ http://www.ccrc.uga.edu/services/
} 
Tobacco roots and cultured cells (Vissenberg et al., 2005) as well as in poplar wood (Nishikubo et al., 2011). XGO-SRs are infiltrated into the tissue and incorporated into xyloglucan in the cell wall yielding an orange fluorescence indicative of the simultaneous colocalization in the same compartment of active XET and acceptor xyloglucan chains. Interestingly, a fibrillar pattern associated with cellulose microfibrils was observed in elongating cells, suggesting that XTHs act on xyloglucans attached to cellulose microfibrils. The XET activity found in elongating cells may play a role in the incorporation of newly synthesized xyloglucan molecules into the cell wall and/or the remodeling of the existing cellulose/xyloglucan network. Glycosyl hydrolase activity also can be visualized in situ. For instance, endo- $\beta$-1,4-xyloglucanase activity was detected in muro using a resorufin $\beta$-glycoside of a xylogluco-oligosaccharide (XXXG- $\beta$-Res; Ibatullin et al., 2009 ) and cellulase activity using a resorufinyl- $\beta$-D-cellobioside (Glc-2- $\beta$-Res; Takahashi et al., 2009).

Another major advance was made with the use of click chemistry to study the metabolism of glycans in vivo. The strategy is based upon the metabolic incorporation of azide sugars into target molecules, priming them for selective covalent reaction with fluorescent probes. The azide is small, stable in biological systems, and selectively reactive with phosphines or activated alkynes. Previously, the Staudinger ligation has been used to tag azido sugar-labeled glycoconjugates on cultured cells and in live mice (Prescher et al., 2004). The copper-catalyzed click chemistry has shown the incorporation of an azido-containing fucose analog into glycoproteins via the fucose salvage pathway in jurkat cells (Sawa et al., 2006). Since these first experiments, the method has been further developed and adapted for plant cells. Anderson et al. (2012) used fucose alkyne (FucAI) to label pectic rhamnogalacturonan-I (RG-I) in roots of Arabidopsis seedlings. Using pulse-chase experiments, they were able to visualize the deposition of pectin and the reorientation of the pectin network during the elongation of epidermal root cells. A problem with this method is that $\mathrm{Cu}(\mathrm{I})$ catalyst formulations are toxic preventing their use in living cells. Different approaches have been developed to overcome this problem. Soriano Del Amo et al. (2010) reported that BTTES, a tris(triazolylmethyl)amine-based ligand for $\mathrm{Cu}(\mathrm{I})$, promotes the cycloaddition reaction rapidly in living systems without apparent toxicity. This catalyst allowed, for the first time, non-invasive imaging of fucosylated glycans during zebrafish early embryogenesis. Recent alternative approaches are based on " $\mathrm{Cu}$-free click chemistry" (for review, see Chang et al., 2010; Jewett and Bertozzi, 2010) to increase the rate of the cycloaddition without the need of a catalyst. These methods have been used to label biomolecules in zebrafish (Laughlin et al., 2008) and in living mice (Chang et al., 2010). It will be extremely interesting to use similar methods to visualize the sites of synthesis, deposition, and turnover of different polysaccharides in plants (Wallace and Anderson, 2012).

\section{FLUORESCENCE MICROSCOPY TO STUDY IN LIVING CELLS THE INTRACELLULAR DYNAMICS AND STOICHIOMETRY OF PROTEIN COMPLEXES INVOLVED IN CELL WALL BIOSYNTHESIS}

Laser scanning confocal microscopy (LSCM) and spinning disk confocal microscopy (SDCM) technologies have been used to study, often in great detail, the dynamics of proteins involved in cell wall synthesis. Whereas LSCM uses a single pinhole for optical sectioning, SDCM uses an array of excitation and emission pinhole apertures (one or two pinhole arrays can be used) on a rapidly spinning disk, such that the pinhole array sweeps the entire field of view over 1,000 times/s. The high scan speed not only improves image acquisition rate (up to $360 \mathrm{frames} / \mathrm{s}$ ), it also has the effect of lowering the peak excitation light density down to a few $\mu \mathrm{W} / \mu \mathrm{m}^{2}$, thereby increasing fluorescence efficiency and decreasing photobleaching and photodamage effects compared to point scanning. Perhaps most importantly, because the entire confocal field of view can be captured by a high-quantum efficiency, low-noise camera instead of a photomultiplier tube (PMT), SDCM systems have a more than 50-fold increase in light capture efficiency (i.e., average number of photons acquired from a single bead plotted against exposure index which is determined by measuring photobleaching rates and not the actual fluorescence at each analyzed location in the specimen), resulting in a several-fold increase in signal-to-noise ratio relative to LSCM. This unexpected difference is accounted for by the large difference in quantum efficiency (percentage of photons hitting the photoreactive surface that will produce an electron-hole pair) between the CCD camera of SDCM (around 60\%) and the PMT of LSCM (around 10\%). However, the reasons for the remainder of the efficiency gap remain unclear (Murray et al., 2007). Gräf etal. (2005) propose an explanation based on the fact that scanning systems, in contrast to SDCM, are often operated at or near saturation for fluorescence, thus a situation in which no new emitted photons are produced while photobleaching increases.

Using SDCM, fluorescent punctae could be observed in the plasma membrane of Arabidopsis hypocotyl cells expressing XFP fused to distinct components of the cellulose synthase complex (CSC; CESA3, CESA6, CESA5, or CSI1/POM2; Paredez et al., 2006; Desprez et al., 2007; Gu et al., 2010; Bischoff et al., 2011; Bringmann et al., 2012; Sanchez-Rodriguez et al., 2012). These punctae migrate bidirectionally following linear trajectories and most likely correspond to individual CSCs. Since the CSCs are propelled by the polymerization of the glucan chains, the rate of the CSCs can be used to quantify cellulose synthesis in vivo. These tools have been extensively used to study the role of cortical microtubules in guiding the CSC trajectories, their insertion into the plasma membrane, the regulation of CSC movement by phosphorylation, as well as the action of small inhibitors of cellulose biosynthesis (DeBolt et al., 2007a,b; Crowell etal., 2009; Gutierrez etal., 2009; Chen etal., 2010; Bischoff et al., 2011).

The intracellular trafficking and the lifetime of proteins can be conveniently studied using photoactivatable or photoconvertible fluorescent proteins (Patterson et al., 2010). Photoactivatable fluorescent proteins have been used in the plant field to analyze the dynamic relationship between the ER and Golgi stacks in tobacco leaf epidermal cells (Runions et al., 2006) or the distribution of KAT1 $\mathrm{K}^{+}$channels in tobacco leaves (Sutter et al., 2006). The property of photoconvertible fluorescent proteins to show pronounced light-induced spectral changes allows pulsechase analysis of protein trafficking. For instance, UV-induced 
green-to-red photoconversion of mEosFP was used to study the translocation of PIN2-mEosFP from one intracellular location to another (Dhonukshe et al., 2007). Such tools will be very useful to analyze the trafficking of CSC components or other cell wall biosynthetic enzymes.

Fluorescence recovery after photobleaching (FRAP) and fluorescence loss in photobleaching (FLIP) are complementary tools for imaging cell wall proteins. Using FRAP, the delivery frequency of CESA complexes to the plasma membrane was estimated at around 5 events $/ \mu \mathrm{m}^{2} \mathrm{~h}$ (Gutierrez et al., 2009). In this case, a short bleaching period followed by post-bleach acquisitions is used to measure any recovery of fluorescence into the bleached area by non-bleached CESA particles. FLIP has been used to study the distribution of CSCs in developing xylem vessels. Using a confocal microscope, yellow fluorescent protein (YFP)-CSCs appear as transverse bands in developing xylem vessels (Wightman and Turner, 2008). To overcome the problem of the high density of YFP-CSCs and the focal depth necessary for imaging deeper layers of the organ, FLIP was used. With this approach, the movement of bleached particles outwards from the bleached region was measured (White and Stelzer, 1999). The identical rates of fluorescence loss on either side of the bleached zone suggested that these CSCs, like the primary cell wall CSCs, also migrate bidirectionally. The motility of YFP-CSC complexes was estimated at around $7 \mu \mathrm{m} / \mathrm{s}$ far higher than those previously reported for primary wall CSCs. It is unlikely, however, that such a high velocity reflects the cellulose synthesis rate (which would be some 14,000 glucose residues/s.chain against 10 residues/s.chain for primary cell walls). It is more likely that the authors have measured the much faster movement of intracellular CSC-containing compartments (Wightman et al., 2009).

Fluorescence microscopy is also commonly used to study protein-protein interactions in plants. Techniques used are fluorescence resonance energy transfer (FRET) or bimolecular fluorescence (BiFC) or luciferase complementation (Jares-Erijman and Jovin, 2003; Bracha-Drori et al., 2004; Fujikawa and Kato, 2007). BiFC strategies have been used to determine specific interactions between the different CESA isoforms (Desprez et al., 2007; Timmers et al., 2009). The N-terminus half of YFP is fused to a first CESA isoform and the C-terminus to a second isoform. Detection of YFP signal signifies protein-protein interaction events since the YFP halves are in sufficient proximity to form an intact YFP molecule. It is an easy and rapid method, however, it is important to note that it creates irreversible interactions and appropriate controls need to be included such as proteins present in the same compartment that do not interact with the tested protein.

A recent improvement in the detection of molecules at the surface of plant cells is the use of total internal reflection fluorescence microscopy (TIRFM). This technique is based on the physical phenomenon of total internal reflection (TIR), which occurs when a ray of light strikes a boundary between two materials with different refractive indices $(n)$ and the incident angle is greater than the critical angle of incidence. Under these conditions, all of the light is reflected back into the medium with the higher $n$ value. When TIR occurs, a near-field light wave forms at the boundary; this "evanescent wave" (EW) can penetrate the surface of the medium to a depth approximately equal to $1 / 3$ of the wavelength of the incident light. The TIRFM can provide around $100 \mathrm{~nm}$ of excitation volume near the plasma membrane. Interestingly despite the presence of the several 100-nm thick cell wall, a TIRF-derived method, known as variable-angle epifluorescence microscopy (VAEM), was recently developed in plants to visualize vesicle trafficking and fusion events at the plasma membrane. The technique uses a laser beam with a suboptimal incident angle, slightly smaller than the critical angle. Konopka et al. were able to visualize the dynamics of membrane proteins, organelles, and cytoskeleton in various plant cells (Konopka and Bednarek, 2008b; Wan et al., 2011) and validated the optical principles underlying VAEM in plant cells. They analyzed in detail the motility of clathrin light chains (CLCs) during endocytosis (Konopka et al., 2008). In both tip growing root hairs and diffusely expanding epidermal cells, a CLC-XFP fusion protein colocalized with the dynaminrelated proteins DRP1C-GFP in dynamic foci at the cell cortex. The authors were able to follow appearance and disappearance at the plasma membrane of the proteins and differentiate the behavior of two distinct DRPs (Konopka and Bednarek, 2008a). The results demonstrate the involvement of DRPs in clathrin-mediated membrane dynamics. Since GFP-CESA3 can be visualized in living cells by VAEM (Figure 1), it will be interesting to see if the internalization of CSCs is also mediated by this pathway. Using VAEM, the organization and dynamics of actin filaments were studied in detail in epidermal cells of Arabidopsis hypocotyls and it has also been shown that there is a gradual appearance of the auxin efflux carrier PIN2-GFP at the outer lateral plasma membrane of root epidermal cells (Staiger et al., 2009; Kleine-Vehn et al., 2011). Interestingly, the VAEM method can also be used to analyze the oligomeric state of proteins. The subunit number and

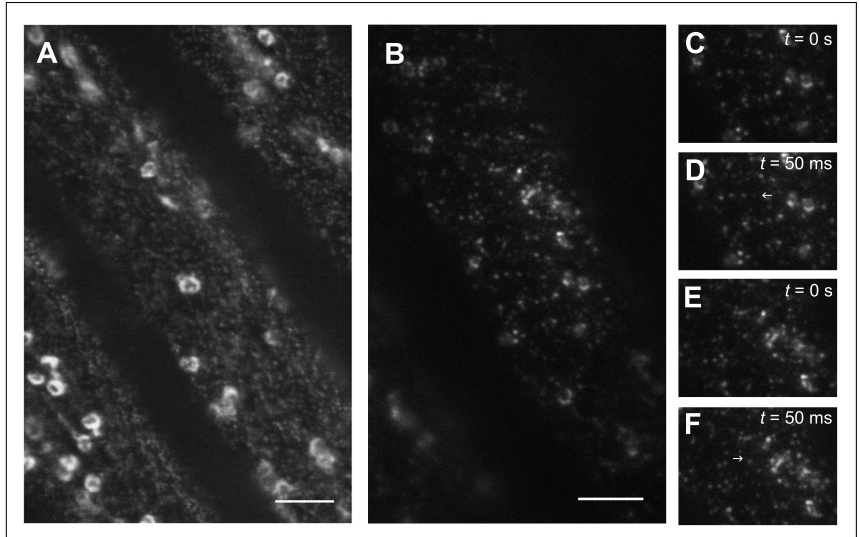

FIGURE 1 | Visualization of GFP-CESA3 using different microscopy techniques. Arabidopsis dark-grown hypocotyl cells expressing the cellulose synthase catalytic subunit CESA3 fused to GFP were imaged using spinning disk confocal microscopy (A) or variable-angle

epifluorescence microscopy (B). Note that in (B), only the vesicles at the cell surface are well defined and thanks to the use of an objective $\times 100$ with a numerical aperture of 1.49 , the xy resolution and the signal-to-noise ratio near the cell edge are improved. Scale bars: $10 \mu \mathrm{M}$. Images from a time series (C-D). Note the appearance of a GFP-CESA3 vesicle in the second image. Images from a time series (E-F). Note the disappearance of a GFP-CESA3 vesicle. Blue arrows indicate the presence or the absence of GFP-CESA3 in successive pictures. 
stoichiometry of membrane-bound proteins can be determined through the statistical analysis of bleaching steps of GFP fused to the proteins. Li et al. (2011) imaged GFP-labeled aquaporin PIP2;1 in the plasma membrane of root cells. The genetic attachment of GFP to PIP2;1 guaranteed that each PIP2;1 polypeptide was labeled with only one fluorophore. To determine the oligomeric state of PIP2;1, they counted the photobleaching steps for chosen fluorescent spots. The maximum number of elemental GFP photobleaching steps in an individual punctum was four, consistent with the aquaporin tetramer observed in crystallographic studies (Tornroth-Horsefield et al., 2006). This approach will be very helpful to study the stoichiometry of cell wall biosynthetic complexes.

Recently, new high-resolution techniques allow imaging beyond the diffraction resolution barrier. Several strategies are being used: stimulated emission depletion (STED) microscopy, linear or non-linear structured illumination microscopy (SIM), and the pointillist techniques: photoactivated localization microscopy (PALM), fluorescence photoactivated localization microscopy (FPALM), and stochastic optical reconstruction microscopy (STORM). While they show great promise for cell wall research, these techniques all have individual strengths and weaknesses. For a detailed discussion, see Leung and Chou (2011). Such methods will be of great value to further analyze the organization of CSCs and their link with cortical microtubules. STED already has been used to show that PIN1 and PIN2 auxin efflux carriers cluster in microdomains in the plasma membrane (Kleine-Vehn et al., 2011).

\section{IMAGING PLANT GROWTH IN 4D}

Major obstacles for 4D (3D + time) imaging are the inherent speed limitation of point-scanning techniques for optical sectioning and the photobleaching that accompanies repeated excitation. The use of light-sheet-based fluorescence microscopy (LSFM), known in different implementations as selective plane illumination (SPIM) or digital scanned laser light-sheet microscopy (DSLM) overcomes

\section{REFERENCES}

Anderson, C. T., Carroll, A., Akhmetova, L., and Somerville, C. (2010). Real-time imaging of cellulose reorientation during cell wall expansion in Arabidopsis roots. Plant Physiol. 152, 787-796.

Anderson, C. T., Wallace, I. S., and Somerville, C. R. (2012). Metabolic click-labeling with a fucose analog reveals pectin delivery, architecture, and dynamics in Arabidopsis cell walls. Proc. Natl. Acad. Sci. U.S.A. 109, 1329-1334.

Bischoff, V., Desprez, T., Mouille, G., Vernhettes, S., Gonneau, M., and Hofte, H. (2011). Phytochrome regulation of cellulose synthesis in Arabidopsis. Curr. Biol. 21, 1822-1827.

Bracha-Drori, K., Shichrur, K., Katz, A., Oliva, M., Angelovici, R., Yalovsky, S., and Ohad, N. (2004). Detection of protein-protein interactions in plants using bimolecular fluorescence complementation. Plant J. 40, 419-427.

Bringmann, M., Li, E., Sampathkumar, A., Kocabek, T., Hauser, M. T., and Persson, S. (2012). POMPOM2/cellulose Synthase Interacting1 is essential for the functional association of cellulose synthase and microtubules in Arabidopsis. Plant Cell 24, 163-177.

Chang, P. V., Prescher, J. A., Sletten, E. M., Baskin, J. M., Miller, I. A., Agard, N. J., Lo, A., and Bertozzi, C. R. (2010). Copper-free click chemistry in living animals. Proc. Natl. Acad. Sci. U.S.A. 107, 1821-1826.

Chen, S., Ehrhardt, D. W., and Somerville, C. R. (2010). Mutations of cellulose synthase (CESA1) phosphorylation sites modulate anisotropic cell expansion and bidirectional mobility of cellulose synthase. Proc. Natl. Acad. Sci. U.S.A. 107, 17188-17193.

these limitations (Huisken et al., 2004; Keller and Stelzer, 2008). The technique is based on the use of a thin sheet of light, which illuminates a single X-Y plane of the specimen, combined with wide-field fluorescence detection with a CCD camera in the direction perpendicular to the excitation plane. With this technique, only a narrow region near the plane of observation is illuminated, as a result fluorophores outside this plane are not excited which reduces background signals and photodamage. These instrumental characteristics produce an optical sectioning effect, improved contrast, and allow long observations of sensitive samples. Threedimensional recording is performed by moving the specimen through the illuminated plane. The acquisition of series of images from three different angles, combined with a processing algorithm, allows the signal fluorescence to be discriminated from the scattered background light. This technique has recently been used to record the growth of vertically oriented primary root tips and lateral root primordia for up to $38 \mathrm{~h}$ without any obvious damage (Maizel et al., 2011). In addition, the dynamics of the endosomal compartments could be visualized for over $40 \mathrm{~s}$ with a speed of 4 frames per second. This technique will allow $3 \mathrm{D}$ reconstructions to be made of entire organs and follow the growth of each individual cell over long time periods.

\section{CONCLUSION}

A large panel of fluorescent imaging techniques is now available for the study of tissues, cells, and cellular components in $3 \mathrm{D}$ or $4 \mathrm{D}$ in plants. These imaging techniques most likely will revolutionize in the coming years our understanding of the dynamics of cell wall architecture and the cellular processes involved in synthesis of cell wall components. However, each imaging modality has relative strengths and weaknesses that need to be taken into account for a given biological problem.

\section{ACKNOWLEDGMENT}

The authors thank Jim Dompierre (Plateforme intégrée Imagif, Gif S/Yvette) for assistance with the VAEM experiments.

Crowell, E. F., Bischoff, V., Desprez, T., Rolland, A., Stierhof, Y. D., Schumacher, K., Gonneau, M., Hofte, H., and Vernhettes, S. (2009). Pausing of Golgi bodies on microtubules regulates secretion of cellulose synthase complexes in Arabidopsis. Plant Cell 21, 1141-1154.

DeBolt, S., Gutierrez, R., Ehrhardt, D. W., Melo, C. V., Ross, L., Cutler, S. R., Somerville, C., and Bonetta, D. (2007a). Morlin, an inhibitor of cortical microtubule dynamics and cellulose synthase movement. Proc. Natl. Acad. Sci. U.S.A. 104, 5854-5859.

DeBolt, S., Gutierrez, R., Ehrhardt, D. W., and Somerville, C. (2007b). Nonmotile cellulose synthase subunits repeatedly accumulate within localized regions at the plasma membrane in Arabidopsis hypocotyl cells following 2,6-dichlorobenzonitrile treatment. Plant Physiol. 145, 334-338.
Desprez, T., Juraniec, M., Crowell, E. F., Jouy, H., Pochylova, Z., Parcy, F., Hofte, H., Gonneau, M., and Vernhettes, S. (2007). Organization of cellulose synthase complexes involved in primary cell wall synthesis in Arabidopsis thaliana. Proc. Natl. Acad. Sci. U.S.A. 104, 15572-15577.

Dhonukshe, P., Aniento, F., Hwang, I., Robinson, D. G., Mravec, J., Stierhof, Y. D., and Friml, J. (2007). Clathrin-mediated constitutive endocytosis of PIN auxin efflux carriers in Arabidopsis. Curr. Biol. 17, 520-527.

Domozych, D. S., Lambiasse, L., Kiemle, S. N., and Gretz, M. R. (2009). Cellwall development and bipolar growth in the Desmid Penium margaritaceum (Zygnematophyceae, Streptophyta). Asymmetry in a symmetric world. $J$. Phycol. 45, 879-893.

Fujikawa, Y., and Kato, N. (2007). Split luciferase complementation assay to 
study protein-protein interactions in Arabidopsis protoplasts. Plant J. 52, 185-195.

Gräf R., Rietdorf, J., and Zimmermann, T. (2005). Live cell spinning disk microscopy. Adv. Biochem. Eng. Biotechnol. 95, 57-75.

Gu, Y., Kaplinsky, N., Bringmann, M., Cobb, A., Carroll, A., Sampathkumar, A., Baskin, T., Persson, S., and Somerville, C. (2010). Identification of a novel CESA-associated protein required for cellulose biosynthesis. Proc. Natl. Acad. Sci. U.S.A. 107, 12866-12871.

Guillen, D., Sanchez, S., and RodriguezSanoja, R. (2010). Carbohydratebinding domains: multiplicity of biological roles. Appl. Microbiol. Biotechnol. 85, 1241-1249.

Gutierrez, R., Lindeboom, J. J., Paredez, A. R., Emons, A. M., and Ehrhardt, D. W. (2009). Arabidopsis cortical microtubules position cellulose synthase delivery to the plasma membrane and interact with cellulose synthase trafficking compartments. Nat. Cell Biol. 11, 797-806.

Huisken, J., Swoger, J., Del Bene, F., Wittbrodt, J., and Stelzer, E. H. (2004). Optical sectioning deep inside live embryos by selective plane illumination microscopy. Science 305, 1007-1009.

Ibatullin, F. M., Banasiak, A., Baumann, M. J., Greffe, L., Takahashi, J., Mellerowicz, E. J., and Brumer, H. (2009). A real-time fluorogenic assay for the visualization of glycoside hydrolase activity in planta. Plant Physiol. 151, 1741-1750.

Jares-Erijman, E. A., and Jovin, T. M. (2003). FRET imaging. Nat. Biotechnol. 21, 1387-1395.

Jewett, J. C., and Bertozzi, C. R. (2010). $\mathrm{Cu}$-free click cycloaddition reactions in chemical biology. Chem. Soc. Rev. 39, 1272-1279.

Keller, P. J., and Stelzer, E. H. (2008). Quantitative in vivo imaging of entire embryos with Digital Scanned Laser Light Sheet Fluorescence Microscopy. Curr. Opin. Neurobiol. 18, 624-632.

Kleine-Vehn, J., Wabnik, K., Martiniere, A., Langowski, L., Willig, K., Naramoto, S., Leitner, J., Tanaka, H., Jakobs, S., Robert, S., Luschnig, C., Govaerts, W., Hell, S. W., Runions, J., and Friml, J. (2011). Recycling, clustering, and endocytosis jointly maintain PIN auxin carrier polarity at the plasma membrane. Mol. Syst. Biol. 7, 540.

Knox, J. P. (2008). Revealing the structural and functional diversity of plant cell walls. Curr. Opin. Plant Biol. 11, 308-313.
Konopka, C. A., Backues, S. K., and Bednarek, S. Y. (2008). Dynamics of Arabidopsis dynamin-related protein $1 \mathrm{C}$ and a clathrin light chain at the plasma membrane. Plant Cell 20, 1363-1380.

Konopka, C. A., and Bednarek, S. Y. (2008a). Comparison of the dynamics and functional redundancy of the Arabidopsis dynamin-related isoforms DRP1A and DRP1C during plant development. Plant Physiol. 147, 1590-1602.

Konopka, C. A., and Bednarek, S. Y. (2008b). Variable-angle epifluorescence microscopy: a new way to look at protein dynamics in the plant cell cortex. Plant J. 53, 186-196.

Laughlin, S. T., Baskin, J. M., Amacher, S. L., and Bertozzi, C. R. (2008). In vivo imaging of membraneassociated glycans in developing zebrafish. Science 320, 664-667.

Lee, K. J., Marcus, S. E., and Knox, J. P. (2011). Cell wall biology: perspectives from cell wall imaging. Mol. Plant 4, 212-219.

Leung, B. O., and Chou, K. C. (2011). Review of super-resolution fluorescence microscopy for biology. Appl. Spectrosc. 65, 967-980.

Li, X., Wang, X., Yang, Y., Li, R., He, Q., Fang, X., Luu, D. T., Maurel, C. and Lin, J. (2011). Single-molecule analysis of PIP2;1 dynamics and partitioning reveals multiple modes of Arabidopsis plasma membrane aquaporin regulation. Plant Cell 23, 3780-3797.

Maizel, A., von Wangenheim, D., Federici, F., Haseloff, J., and Stelzer, E. H. (2011). High-resolution live imaging of plant growth in near physiological bright conditions using light sheet fluorescence microscopy. Plant J. 68, 377-385.

Marcus, S. E., Blake, A. W., Benians, T. A., Lee, K. J., Poyser, C., Donaldson, L., Leroux, O., Rogowski, A., Petersen, H. L., Boraston, A., Gilbert, H. J., Willats, W. G., and Knox, J. P. (2010). Restricted access of proteins to mannan polysaccharides in intact plant cell walls. Plant J. 64, 191-203.

Marcus, S. E., Verhertbruggen, Y., Herve, C., Ordaz-Ortiz, J. J., Farkas, V., Pedersen, H. L., Willats, W. G., and Knox, J. P. (2008). Pectic homogalacturonan masks abundant sets of xyloglucan epitopes in plant cell walls. BMC Plant Biol. 8, 60. doi: 10.1186/1471-2229-8-60

Moller, I., Marcus, S. E., Haeger, A., Verhertbruggen, Y., Verhoef, R., Schols, H., Ulvskov, P., Mikkelsen, J. D., Knox, J. P., and Willats, W. (2008). High-throughput screening of monoclonal antibodies against plant cell wall glycans by hierarchical clustering of their carbohydrate microarray binding profiles. Glycoconj. J. 25, 37-48.

Murray, J. M., Appleton, P. L., Swedlow, J. R., and Waters J. C. (2007). Evaluating performance in three-dimensional fluorescence microscopy. J. Microsc. 228, 390-405.

Nishikubo, N., Takahashi, J., Roos, A. A., Derba-Maceluch, M., Piens, K., Brumer, H., Teeri, T. T., Stalbrand, H., and Mellerowicz, E. J. (2011). Xyloglucan endo-transglycosylasemediated xyloglucan rearrangements in developing wood of hybrid aspen. Plant Physiol. 155, 399-413.

Paredez, A. R., Somerville, C. R., and Ehrhardt, D. W. (2006). Visualization of cellulose synthase demonstrates functional association with microtubules. Science 312, 1491-1495.

Pattathil, S., Avci, U., Baldwin, D., Swennes, A. G., McGill, J. A., Popper, Z., Bootten, T., Albert, A., Davis, R. H., Chennareddy, C., Dong, R., O'Shea, B., Rossi, R., Leoff, C., Freshour, G., Narra, R., O’Neil, M., York, W. S., and Hahn, M. G. (2010). A comprehensive toolkit of plant cell wall glycan-directed monoclonal antibodies. Plant Physiol. 153, 514-525.

Patterson, G., Davidson, M., Manley, S., and Lippincott-Schwartz J. (2010). Superresolution imaging using single-molecule localization. Annu. Rev. Phys. Chem. 61, 345-367.

Prescher, J. A., Dube, D. H., and Bertozzi, C. R. (2004). Chemical remodelling of cell surfaces in living animals. Nature 430, 873-877.

Ralet, M. C., Tranquet, O., Poulain, D., Moise, A., and Guillon, F. (2010). Monoclonal antibodies to rhamnogalacturonan I backbone. Planta 231, 1373-1383.

Roelofsen, P. A., and Houwink, A. L. (1953). Architecture and growth of the primary cell wall in some plant hairs and in the Phycomyces sporangiophore. Acta Bot. Neerland 2, 218-225.

Runions, J., Brach, T., Kuhner, S., and Hawes, C. (2006). Photoactivation of GFP reveals protein dynamics within the endoplasmic reticulum membrane. J. Exp. Bot. 57, 43-50.

Sanchez-Rodriguez, C., Bauer, S., Hematy, K., Saxe, F., Ibanez, A. B., Vodermaier, V., Konlechner, C., Sampathkumar, A., Ruggeberg, M., Aichinger, E., Neumetzler, L., Burgert, I., Somerville, C., Hauser, M.
T., and Persson, S. (2012). Chitinaselikel/pom-poml and its homolog CTL2 are glucan-interacting proteins important for cellulose biosynthesis in Arabidopsis. Plant Cell 24, 589-607.

Sawa, M., Hsu, T. L., Itoh, T., Sugiyama, M., Hanson, S. R., Vogt, P. K., and Wong, C. H. (2006). Glycoproteomic probes for fluorescent imaging of fucosylated glycans in vivo. Proc. Natl. Acad. Sci. U.S.A. 103, 12371-12376.

Soriano Del Amo, D., Wang, W., Jiang, H., Besanceney, C., Yan, A. C., Levy, M., Liu, Y., Marlow, F. L., and Wu, P. (2010). Biocompatible copper(I) catalysts for in vivo imaging of glycans. J. Am. Chem. Soc. 132, 16893-16899.

Staiger, C. J., Sheahan, M. B., Khurana, P., Wang, X., McCurdy, D. W., and Blanchoin, L. (2009). Actin filament dynamics are dominated by rapid growth and severing activity in the Arabidopsis cortical array. J. Cell Biol. 184, 269-280.

Sutter, J. U., Campanoni, P., Tyrrell, M., and Blatt, M. R. (2006). Selective mobility and sensitivity to SNAREs is exhibited by the Arabidopsis KAT1 $\mathrm{K}^{+}$channel at the plasma membrane. Plant Cell 18, 935-954.

Takahashi, J., Rudsander, U. J., Hedenstrom, M., Banasiak, A., Harholt, J., Amelot, N., Immerzeel, P., Ryden, P., Endo, S., Ibatullin, F. M., Brumer, H., del Campillo, E., Master, E. R., Scheller, H. V., Sundberg, B., Teeri, T. T., and Mellerowicz, E. J. (2009). KORRIGAN1 and its aspen homolog PttCel9A1 decrease cellulose crystallinity in Arabidopsis stems. Plant Cell Physiol. 50, 1099-1115.

Timmers, J., Vernhettes, S., Desprez, T., Vincken, J. P., Visser, R. G., and Trindade, L. M. (2009). Interactions between membrane-bound cellulose synthases involved in the synthesis of the secondary cell wall. FEBS Lett. $583,978-982$.

Tornroth-Horsefield, S., Wang, Y., Hedfalk, K., Johanson, U., Karlsson, M., Tajkhorshid, E., Neutze, R., and Kjellbom, P. (2006). Structural mechanism of plant aquaporin gating. Nature 439, 688-694.

Vissenberg, K., Fry, S. C., Pauly, M., Hofte, H., and Verbelen, J. P. (2005). XTH acts at the microfibril-matrix interface during cell elongation. J. Exp. Bot. 56, 673-683.

Wallace, I., and Anderson, C. T. (2012). Small molecule probes for plant cell wall polysaccharide imaging. Front. Plant Physiol. 3:89. doi: 10.3389/fpls.2012.00089

Wan, Y., Ash, W. M. III., Fan, L., Hao, H., Kim, M. K., and Lin, J. (2011). Variable-angle total internal 
reflection fluorescence microscopy of intact cells of Arabidopsis thaliana. Plant Methods 7, 27.

White, J., and Stelzer, E. (1999). Photobleaching GFP reveals protein dynamics inside live cells. Trends Cell Biol. 9, 61-65.

Wightman, R., Marshall, R., and Turner, S. R. (2009). A cellulose synthasecontaining compartment moves rapidly beneath sites of secondary wall synthesis. Plant Cell Physiol. 50, 584-594.

Wightman, R., and Turner, S. R. (2008).

The roles of the cytoskeleton during cellulose deposition at the secondary cell wall. Plant J. 54, 794-805.

Conflict of Interest Statement: The authors declare that the research was conducted in the absence of any commercial or financial relationships that could be construed as a potential conflict of interest.

Received: 15 March 2012; accepted: 13 June 2012; published online: 03 July 2012.

Citation: Gonneau $M$, Höfte $H$ and Vernhettes $S$ (2012) Fluorescent tags to explore cell wall structure and dynamics. Front. Plant Sci. 3:145. doi: 10.3389/ fpls.2012.00145
This article was submitted to Frontiers in Plant Physiology, a specialty of Frontiers in Plant Science.

Copyright (c) 2012 Gonneau, Höfte and Vernhettes. This is an open-access article distributed under the terms of the Creative Commons Attribution Non Commercial License, which permits noncommercial use, distribution, and reproduction in other forums, provided the original authors and source are credited. 